\title{
Field Applications of Fiber-Optic Sensors. Part I: Temperature Measurements in a Geothermal Well
}

\author{
S. M. ANGEL, ${ }^{*}$ D. G. GARVIS, S. K. SHARMA, and A. SEKI \\ Environmental Sciences Division, Lawrence Livermore National Laboratory, Livermore, California 94550 (S.M.A., D.G.G.); \\ and Hawaii Institute of Geophysics (S.K.S.) and Hawaii Natural Energy Institute (A.S.), University of Hawaii, \\ Honolulu, Hawaii 96822
}

\begin{abstract}
We have initiated a program for developing and field testing fiber-opticsbased sensors to monitor in situ physical and chemical parameters in highly corrosive environments, such as geothermal wells, oil wells, and hot-water boiler reactors. Inability to sample hot geothermal wells or to measure the chemical composition of hot brines limits our understanding of $i n$ situ conditions in geothermal fields. In this communication, we report preliminary results obtained with a temperature optrode to profile the temperature in a geothermal steam well. To our best knowledge, this is the first time in situ geothermal well measurements have been made with the use of a fiber-optic sensor.
\end{abstract}

Index Headings: Fluorescence; Luminescence; Fiber-optic sensors; Spectroscopic techniques.

\section{INTRODUCTION}

While technology has advanced in certain aspects of geothermal exploration, instrumentation for downhole measurements has not advanced significantly. The present methods for gathering temperature, pressure, and water-chemistry information about the fluids in hightemperature wells include measurement by mechanical instruments and by electronic instruments. There are limitations to each of these methods. ${ }^{1}$ Some of the limits of mechanical instruments are that (1) results are not provided on a timely basis because most chemical concentrations are determined on a batch sampling basis in laboratories removed from the site, and results are not available for a few hours or a few days; (2) results for water chemistry are suspect because water from another depth may leak into the samples; and (3) immediate changes in constituents and concentrations generally cannot be assessed, because only a limited number of chemical compounds can be measured in real time, and those measurements can only be made under restricted conditions. Present downhole data acquisition by electronic instruments is limited because heavy conducting

\footnotetext{
Received 3 November 1988.

* Author to whom correspondence should be sent.
}

cables are required and there is a high incidence of instrument failure at high temperatures.

Real-time downhole measurements are essential in the development of geothermal wells and fields. The variation of the temperature in the well as a function of depth and of time is important in determining the source of the hot water in the well and for predicting the future production of the well. These parameters, in addition to water chemistry in the well, are important in determining the useful life of a well. Furthermore, understanding the water chemistry in a well is critical for designing equipment that is resistant to scaling and can withstand corrosive fluids.

Recently, a program was started at Lawrence Livermore National Laboratory (LLNL) to develop opticalfiber sensors (optrodes) for use in logging and monitoring geothermal wells. Research was focused on developing a temperature optrode that could be used to obtain realtime information at temperatures up to $300^{\circ} \mathrm{C}$ in very deep geothermal wells. This optrode was completed and has been thoroughly tested in the laboratory. The most serious limitation of this optrode is that the optical fiber must be kept dry at high temperatures.

Optical-fiber temperature measurements at LLNL are based on the temperature-dependent fluorescence characteristics of inorganic ions doped into a host matrix. A ruby-based temperature and pressure optrode that operates in the temperature range of $100^{\circ}$ to $600^{\circ} \mathrm{C}$ has been reported previously. ${ }^{2}$ The ruby-based optrode has a temperature accuracy that is very dependent on the temperature range being measured and is better at higher temperatures. At $400^{\circ} \mathrm{C}$ the accuracy is about 1 to $2^{\circ} \mathrm{C} .^{3}$ More recently, a temperature optrode has been developed that is based on dual-emission rare-earth phosphorescence. ${ }^{4-6}$ The new optrode has the advantage of working over a wider temperature range and in corrosive environments, and it uses simpler instrumentation. Further, it has an improved accuracy because the intensity ratio of two emission bands is measured. The objective 

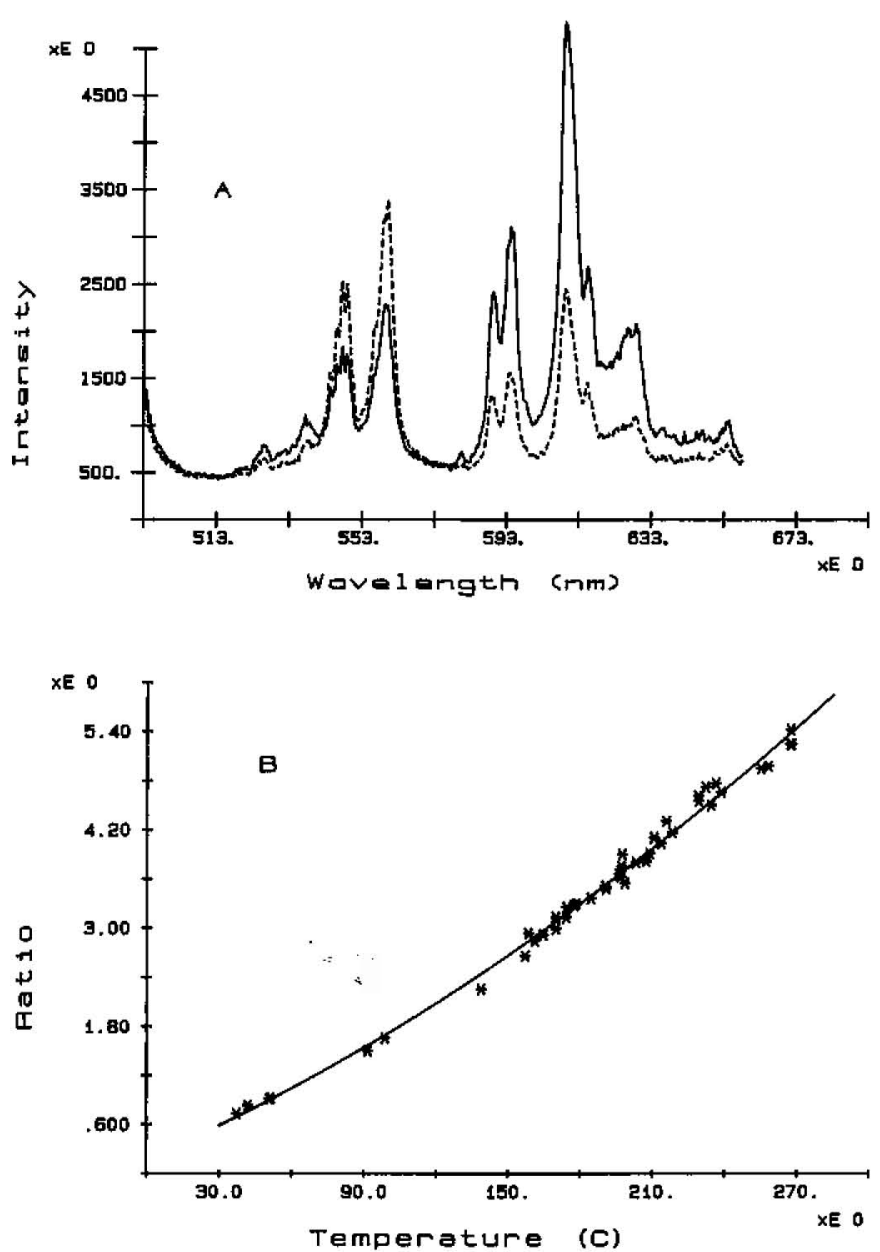

Fig. 1. Single-fiber temperature optrode response with the use of a short (2-m) optical fiber: (A) the optrode fluorescence response at $37.3^{\circ} \mathrm{C}$ (dashed) and $138^{\circ} \mathrm{C}$ (solid); (B) response curve for the temperature optrode with the use of a 2-m optical fiber, plotted as the ratio of the intensities of the $\mathrm{Eu}^{+3}$ and the $\mathrm{Er}^{+3}$ fluorescence peaks, versus temperature.

of the research reported here is to demonstrate that a temperature optrode can be used to obtain real-time information from a geothermal well and that it can be used interactively.

\section{EXPERIMENTAL}

The new temperature optrode is based on the temperature-dependent phosphorescence of the $\mathrm{Eu}^{+3}$ ion. Briefly, $\mathrm{Eu}^{+3}$ and $\mathrm{Er}^{+3}$ are doped into lattice sites within an inert ceramic-like carrier matrix $\left(\mathrm{CaZrTi}_{2} \mathrm{O}_{7}\right)$. The two dopants both fluoresce when excited with the 488$\mathrm{nm}$ line of an argon-ion laser. In the case of $\mathrm{Eu}^{3+}$, the intensity of the fluorescence bands at 610 and $595 \mathrm{~nm}$ increases as the temperature increases, while, in the case of $\mathrm{Er}^{3+}$, the intensity of the fluorescence bands at 548 and $560 \mathrm{~nm}$ decreases as the temperature increases (plot A of Fig. 1). The ratio of the $\mathrm{Eu}^{3+}$ and $\mathrm{Er}^{3+}$ band intensities is plotted as a function of temperature to produce a calibration curve for the optrode (plot B of Fig. 1). The accuracy of this optrode is about $\pm 0.2^{\circ} \mathrm{C}$ in the range 100 to $300^{\circ} \mathrm{C}$, with the use of a short (1 to $2 \mathrm{~m}$ ) optical fiber and a laboratory spectrometer. Of course, the accuracy of the sensor is related to the type of spectrometer used

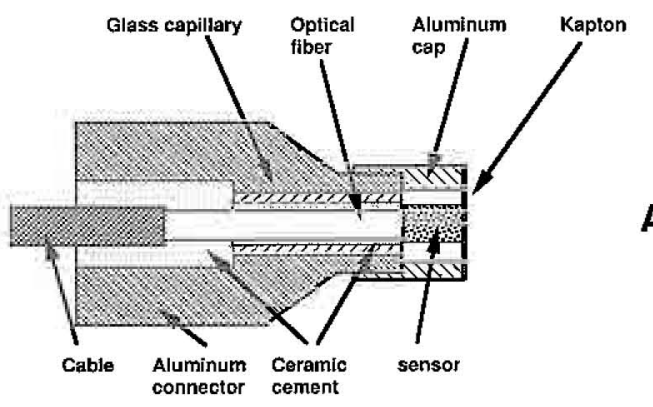

A)

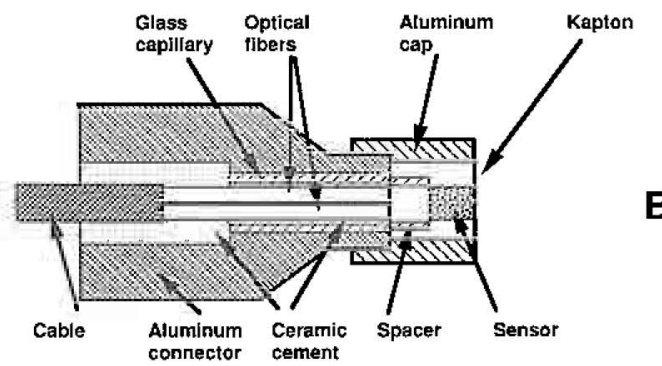

Fiç. 2. Optrode construction: (A) single-fiber optrode construction; (B) double-fiber optrode construction.

to make the measurement, the laser power, and many other parameters that vary from spectrometer to spectrometer.

The details of the optrode construction are shown in Fig. 2. The optrode was made by modifying a standard optical-fiber connector (Ofti-brand, $250-\mu \mathrm{m}$ hole) to hold the temperature-sensitive ceramic. The ceramic was machined into a $3-\mathrm{mm}$-long cylinder about $2 \mathrm{~mm}$ in diameter. For the single-fiber measurements, we used a temperature-resistant film (Kapton) on one flat end of the ceramic cylinder to hold the ceramic piece tight against the polished face of the optical fiber (Fig. 2A). The single fiber was glued in the optical-fiber connector with a temperature-resistant ceramic cement (Cotronics Corporation, No. 940, zirconia-based adhesive), and this optical fiber was used both to illuminate the sensor and to collect the resulting ffuorescence signal. All well measurements were made over a single, $300-\mathrm{m}$ length of high-temperature, polyimide-coated, fused-silica optical fiber (Polymicro Incorporated FHP $320 / 385 / 415$ ) with a $320-\mu \mathrm{m}$ core diameter.

The double-fiber probe was $100-\mathrm{m}$ long and used two $200-\mu \mathrm{m}$-core-diameter optical fibers (Diaguide brand 200/ 250 glass on glass optical fiber). The optrode was constructed as described above, with the exception that a 2-mm spacer was used to separate the temperature-sensitive ceramic from the end of the two optical fibers (Fig. $2 B$ ). This configuration permitted good overlap between the fields of view of the two optical fibers at the optrode end, thus providing optimal collection of the fluorescence generated in the optrode. The two fibers were held in a single Ofti connector with minimum separation between them.

The field spectrometer setup is shown in Fig. 3. The 488-nm line from an air-cooled argon-ion laser was used 


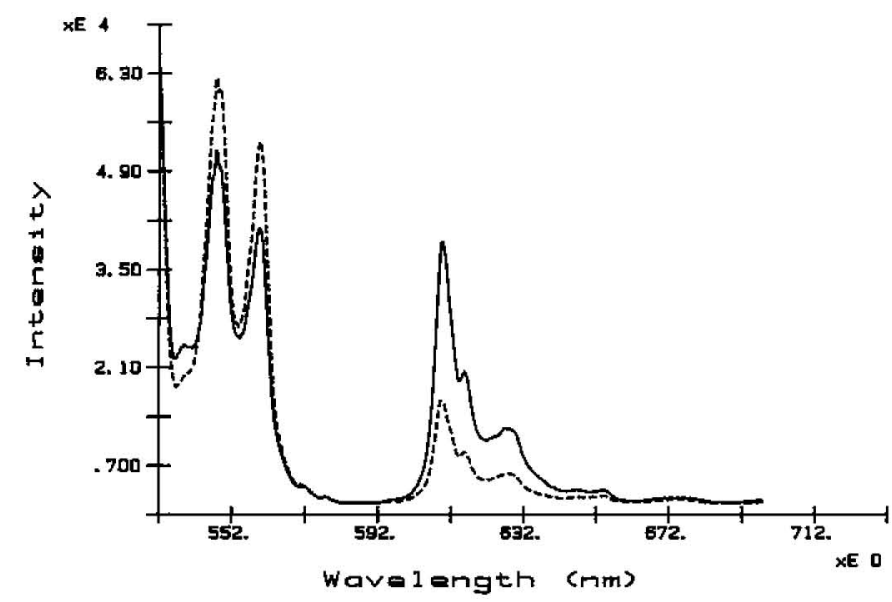

FIG. 4. Single-fiber temperature optrode response with the use of a $300-\mathrm{m}$ optical fiber and a narrow-band rejection filter to attenuate the Raman band at $600 \mathrm{~nm}$ : (dashed) $25.5^{\circ} \mathrm{C}$; (solid) $85^{\circ} \mathrm{C}$.

the narrow-band rejection filter; this filter also blocks the 595-nm $\mathrm{Eu}^{+3}$ emission band. The calibration was done in the dark and without subtracting the Raman background component of the optrode signal. A 38-L temperature-controlled water bath was used for the calibration; the temperature calibration range was between 31.8 and $91.0^{\circ} \mathrm{C}$. The water temperature, as measured by a $\mathrm{K}$-type thermocouple, varied by $\pm 2^{\circ} \mathrm{C}$ at different positions in the bath, and this variation limited the accuracy of the optrode calibration. Optrode measurements were made while the temperature of the water bath was increased from 31.8 to $91.0^{\circ} \mathrm{C}$ and again as the water bath cooled back down to room temperature. In Fig. 5 is shown the resulting calibration curve. The solid curve in Fig. 5 represents a polynomial fit of the optrode response. The polynomial equation was used to relate the optrode response in the well to the temperature in the well.

The well measurements were made by lowering the temperature optrode to a particular depth while monitoring the real-time response of the detector on an os-

$\ddagger$ The polynomial equation used to fit the temperature optrode response is: Response $=0.4684+(0.0069 * T)+\left(1.57 * 10^{-5 *} T^{2}\right)$.

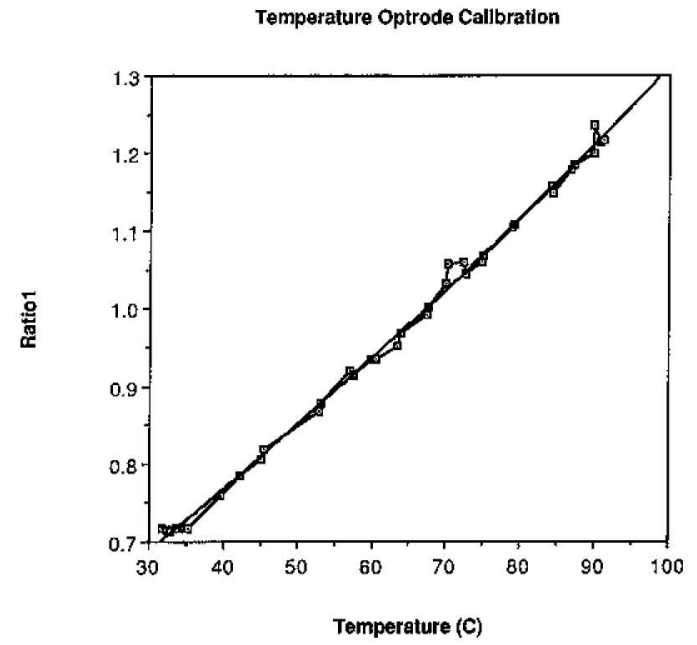

Fig. 5. Calibration curve from 31.8 to $91.0^{\circ} \mathrm{C}$ with the use of a $300-\mathrm{m}$ optical fiber and the single-fiber optrode. The solid line shows the polynomial curve fit to the calibration data as described in the text.

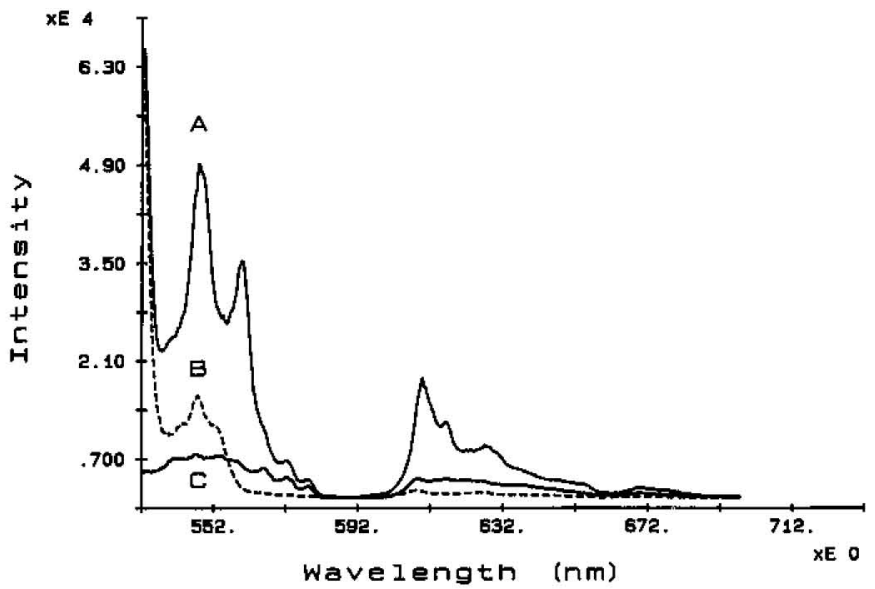

FIG. 6. Fluorescence response of the temperature optrode, at a depth of $15.2 \mathrm{~m}$ in the well, showing the Raman and sunlight contributions to the total signal. (A) The total signal comprised of the optrode fluorescence signal, the Raman background, and the sunlight contributions; (B) the Raman contribution obtained by removing the optrode from the fiber with the fiber in the dark; (C) the sunlight contribution obtained by shuttering the excitation laser.

cilloscope. This approach was found to be very useful for quickly locating and examining interesting spots in the well. After the probe was positioned to a particular depth, the sensor response was allowed to reach equilibrium (almost immediately), and three spectra were measured. One spectrum was measured with the sensor illuminated (fluorescence signal plus background signal), followed by one with the laser shuttered (background signal only), and a second spectrum with the sensor illuminated. The shuttered spectrum was used for subtracting the variable background signal that was due to sunlight leaking into the optical fiber. A thermocouple reading was also recorded at each depth. After the measurements were completed at a particular depth, the probe was repositioned and the procedure was repeated. Measurements were taken as the probe was lowered down the well as well as when it was raised back to the surface. When results from a region appeared to be interesting, measurements were repeated several times. In this way, a depth vs. temperature profile (well $\log$ ) was obtained very quickly.

In Fig. 6 are shown spectra that were measured with the fiber in the well at a depth of $15.2 \mathrm{~m}$ (50 ft), including the Raman and sunlight components. In plot A of Figure 6 is shown the spectrum that results from the combination of optrode fluorescence, Raman signal generated in the optical fiber, and sunlight background. Plot B of Fig. 6 was obtained by removing the optrode and measuring the signal that resulted only from illumination of the optical fiber. This spectrum was measured with the optical fiber in the dark and is due only to the Raman signal generated in the optical fiber. Finally, the spectrum shown in plot C of Fig. 6 was obtained by shuttering the laser and is due only to the sunlight background component.

The temperature in the well is determined by subtracting plot C of Fig. 6 (sunlight background) from plot A of Fig. 6 (Optrode signal plus Raman signal plus sunlight background) and calculating the ratio of the $\mathrm{Eu}^{3+}$ band intensity at $610 \mathrm{~nm}$ and the $\mathrm{Er}^{3+}$ band intensity at $560 \mathrm{~nm}$ from the resulting spectrum. The ratio is then 
trode. It was shown that the Raman background can be greatly reduced by using a dual-fiber optrode-only, however, at the expense of greatly reduced signal levels.

\section{FUTURE DIRECTIONS}

In the future, other types of optrodes will be tested to measure chemical parameters in geothermal wells. Hightemperature $\mathrm{pH}$ and $\mathrm{Eh}$ optrodes are already being tested for this purpose. In the more immediate future, the temperature optrode will be tested in hotter and deeper geothermal wells. It has been tested in the laboratory to $320^{\circ} \mathrm{C}$ at several thousand-psi pressure for extended periods of time. However, to be used at such high temperatures, the optical fiber has to be well shielded and kept from directly contacting the well fluids. On the basis of the magnitude of the fluorescence signal generated by the optrode with the use of 300 meters of optical fiber and the known attenuation of the optical fiber, it is believed that wells up to $1000 \mathrm{~m}$ in depth can be logged. With the use of the present instrument configuration, spectra can be obtained at a rate of about 30 per second. This will allow very high spatial resolution measurements to be made as the probe is continuously lowered into the well.

\section{ACKNOWLEDGMENTS}

The work at Lawrence Livermore National Laboratory was performed under the auspices of the U.S. Department of Energy under Contract W-7405-Eng-48. Additional funding was provided by the $\mathrm{Ha}$ waii Natural Energy Institute (HNEI). The authors would like to ex- press thanks to Dr. Marshall Reed of DOE and Dr. Paul Kasameyer of LLNL for their interest and support of this work. We would also like to thank Dr. Ken Marsh for furnishing the laser that was used in these experiments and Dr. Patrick Takahashi of HNEI for his encouragement. Also, thanks to Mr. Jack Huizingh and Roy Nakanishi of the Natural Energy Laboratory of Hawaii for use and access to the Puna Research Center and to Mr. Don Heinzen of Amfac for access to GTW-2. In addition, we express thanks to Mr. Ben Respicio of the University of Hawaii engineering shop for fabrication of the fiber-optic line pulley stand and to Dr. Tom Kulp of LLNL for his role in the experiments.

1. M. D. Lamers, Measurement Requirements and Methods for Geothermal Reservoir System Parameters (an Appraisal), Technical Report LBL-9090/GREMP-61UC-66b (Lawrence Berkeley Laboratory, Berkeley, California, 1979).

2. G. Haugen, and T. Hirschfeld, Fiber Optics Temperature and Pressure Probe, UCID-20563, (Lawrence Livermore National Laboratory, Livermore, California, 1985).

3. G. Haugen, Staff Scientist, Lawrence Livermore National Laboratory, Livermore, California, personal communication.

4. S. M. Angel, 1986 Pittsburgh Conference and Exposition, Atlantic City, New Jersey (1986), Paper No. 673.

5. S. M. Angel, Geothermal Resources Council, Transactions 11, 155 (1987).

6. S. M. Angel and T. Hirschfeld, "Dual-Emission Rare-Earth Temperature Optrode," manuscript in preparation.

7. D. Nakano, Division of Water and Land Development, Dept. of Land and Natural Resources, Honolulu, Hawaii, personal communication,

8. D. Epp, and A. J. Halunen, Jr., "Temperature Profiles in Wells on the Island of Hawaii," in Geothermal Resources Exploration in Hawaii: Series No. 7 (Hawaii Institute of Geophysics, University of Hawaii, Honolulu, 1979).

9. D. Thomas, "A Geochemical Model of the Kilauea East Rift Zone," in Volcanism in Hawaii, USGS Professional Paper (United States Geological Survey, 1987), Vol. II, pp. 1507-1525. 